\title{
Effects of collision energy on stereodynamical information of the reaction $\mathbf{F}+\mathbf{H I} \rightarrow \mathbf{F I}+\mathbf{H}$
}

\author{
JIAN-JUN MA \\ Department of Applied Physics, Anhui University of Technology, Maanshan 243032, China \\ e-mail: mjjdlut@163.com
}

MS received 18 February 2015; revised 24 May 2015; accepted 27 May 2015

\begin{abstract}
The vector correlations between products and reactants for the reaction $\mathrm{F}+\mathrm{HI} \rightarrow \mathrm{FI}+\mathrm{H}$ are studied using quasi-classical trajectory (QCT) method. Employing a semiempirical LEPS potential energy surface constructed by Kornweitz and Persky, this work calculates the angular distributions of $P\left(\theta_{r}\right), P\left(\phi_{r}\right)$ and several PDDCSs in the center of mass frame. The effect of the collision energy on the product vector properties is also discussed.
\end{abstract}

Keywords. Quasiclassical trajectory; vector correlations; stereodynamics; F+HI.

\section{Introduction}

The reaction between $\mathrm{F}$ atom and $\mathrm{HI}$ molecule can proceed via the following two reactive paths,

$$
\begin{gathered}
\mathrm{F}+\mathrm{HI} \rightarrow \mathrm{HF}+\mathrm{I} \\
\rightarrow \mathrm{IF}+\mathrm{H}
\end{gathered}
$$

The first path is of much interest and importance in relation to chemical lasers due to the highly vibrational excited HF product. The experimental and theoretical researches for the first path mainly involve the vibrational distributions of $\mathrm{HF}$ and determination of the rate constants. From the experimental viewpoint, various experimental methods were used in the studies of the vibrational distribution of the product HF. Population inversion of the vibrational states of the HF was found. ${ }^{1-3}$ Würzberg and Houston ${ }^{4}$ measured the rate constant in the temperature range from 195 to $373 \mathrm{~K}$ through laser photo-dissociation and infrared fluorescence techniques and found a nonArrhenius temperature dependence for this reaction. Theoretically, based on semiempirical London-EyringPolanyi-Sato(LEPS) potential energy surface (PES), Beadle et $a l^{2}{ }^{2}$ calculated the rate constant and the vibrational state population of HF for the first path by the QCT method. But there exists an obvious difference between theoretical results and experimental data. In 2004, Kornweitz and Persky ${ }^{5}$ constructed a new LEPS $\mathrm{PES}$ for the reactions $\mathrm{F}+\mathrm{HCl}, \mathrm{F}+\mathrm{HBr}$ and $\mathrm{F}+\mathrm{HI}$ using new spectral data and carried out dynamical calculation by QCT method. Their calculated results about the first reaction rate constants, energy distribution and vibrational population of HF are in good agreement with experimental data. Most recently, employing this new LEPS PES, Mu and Duan ${ }^{6}$ studied the influence of collision energy and rotational excitation of reactant molecules on stereodynamics for the first the reactive path through QCT method. However, to the best of our knowledge, the existing work on the $\mathrm{F}+\mathrm{HI}$ reaction mainly focuses on the first reactive path, while there are only a few reports about the second reactive path due to its higher energy barrier (about $6 \mathrm{kcal} / \mathrm{mol}$ ). As to this kind of reaction systems, the product rotational angular momentum is strongly aligned about the direction of the relative velocity of the reactant. In the present work we focus our attention mainly on the effect of the collision energy on stereodynamics of the reaction $\mathrm{F}+\mathrm{HI} \rightarrow \mathrm{FI}+\mathrm{H}$, expecting to make some new discovery.

\section{Theory}

The general theory of the product rotational polarization and calculating method of the QCT is the same as that reported..$^{7-22}$ Only the details relevant to the present work are summarized here. The center-of-mass reference frame is shown in figure 1 . The $\mathrm{z}$ axis is parallel to initial relative velocity vector $\boldsymbol{k}$, and the y axis is perpendicular to the $\mathrm{x}-\mathrm{z}$ scattering plane containing the initial and final relative velocity vectors, $\boldsymbol{k}$ and $\boldsymbol{k}^{\prime} . \theta_{t}$ is the scattering angle (i.e. the angle between $\boldsymbol{k}$ and $\boldsymbol{k}^{\prime}$ ); $\theta_{r}$ and $\phi_{r}$ are the polar and azimuthal angle of the final rotational angular momentum $\boldsymbol{j}^{\prime}$, respectively.

The total reaction cross-section is defined as

$$
\sigma_{r}=\pi b_{\max }^{2} \frac{N_{r}}{N_{T}},
$$




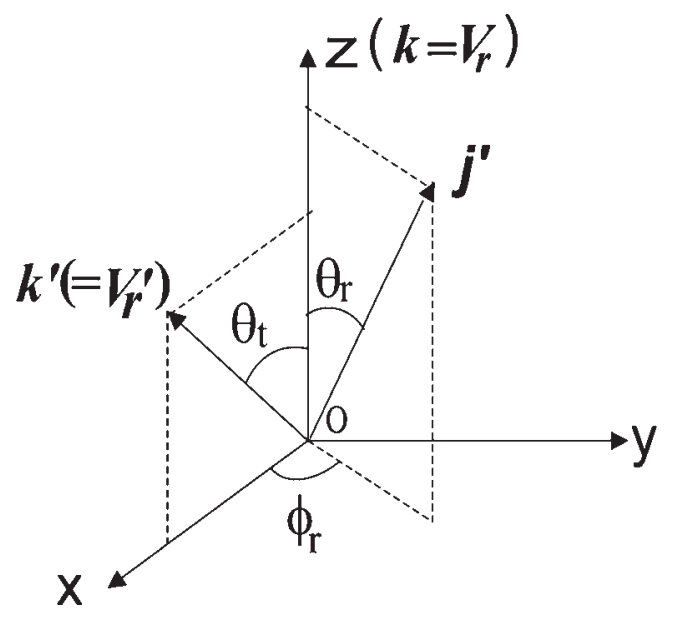

Figure 1. Center of mass frame used to describe the $\boldsymbol{k}, \boldsymbol{k}^{\prime}$ and $j^{\prime}$ correlations.

where $N_{r}$ is the number of reactive trajectories and $N_{T}$ is the total number of trajectories.

The fully correlated center-of-mass angular distribution can be expressed as

$$
P\left(\omega_{t}, \omega_{r}\right)=\sum_{k q} \frac{2 k+1}{4 \pi} P_{k q}\left(\omega_{t}\right) C_{k q}\left(\theta_{r}, \phi_{r}\right)^{*}
$$

where $C_{k q}\left(\theta_{r}, \phi_{r}\right)=\sqrt{4 \pi /(2 k+1)} Y_{k q}\left(\theta_{r}, \phi_{r}\right)$ is the modified spherical harmonics. And $P_{k q}\left(\omega_{t}\right)$ denotes a generalized polarization-dependent differential cross section (PDDCS), which is defined as

$$
P_{k q}\left(\omega_{t}\right)=\frac{1}{\sigma} \frac{d \sigma_{k q}}{d \omega_{t}}=\int P\left(\omega_{t}, \omega_{r}\right) C_{k q}\left(\theta_{r}, \phi_{r}\right) d \omega_{r}
$$

where $d \omega_{r}=\sin \theta_{r} d \theta_{r} d \phi_{r}$. When $q \neq 0, P_{k q}\left(\omega_{t}\right)$ are complex spherical tensor operators. The real $P_{k q}\left(\omega_{t}\right)$ with $q \neq 0$ are defined as

$$
\begin{aligned}
P_{k q \pm}\left(\omega_{t}\right)= & \frac{1}{\sqrt{2}} i^{(-1 \pm 1)}\left[P_{k q}\left(\omega_{t}\right) \pm P_{k-q}\left(\omega_{t}\right)\right] \\
= & \frac{1}{\sqrt{2}} i^{(-1 \pm 1)}(-1)^{q}\left[1 \pm(-1)^{k}\right] \\
& \times \sum_{k_{1} \geq q} \frac{2 k+1}{4 \pi} s_{k q}^{k_{1}} C_{k_{1}-q}\left(\theta_{t}, 0\right)
\end{aligned}
$$

with

$$
s_{k q}^{k_{1}}=(-1)^{q}\left\langle C_{k_{1} q}\left(\theta_{t}, 0\right) C_{k q}\left(\theta_{r}, 0\right) \exp \left(i q \phi_{r}\right)\right\rangle
$$

where the brackets indicate averaging over all the trajectories.

$P_{k q \pm}\left(\omega_{t}\right)$ with $(k \neq 0, q \neq 0)$ adopt the renormalized form,

$$
\tilde{P}_{k q \pm}\left(\omega_{t}\right)=P_{k q \pm}\left(\omega_{t}\right) / P_{00}\left(\omega_{t}\right)
$$

Table 1. The maximum impact parameters $\left(b_{\max }\right)$, reaction probability $\left(N_{r /} N_{T}\right)$ and total reaction cross-section for each trajectory at a given collision energy (Ecol).

\begin{tabular}{lcccc}
\hline $\mathrm{E}_{\mathrm{col}}(\mathrm{eV})$ & 0.4 & 0.7 & 1.0 & 1.5 \\
$\mathrm{~b}_{\max }(\hat{A})$ & 1.932 & 2.024 & 2.116 & 2.116 \\
$\mathrm{~N}_{\mathrm{r}} / \mathrm{N}_{\mathrm{T}}(\%)$ & 16.96 & 24.50 & 27.33 & 32.84 \\
$\sigma_{r}\left(\AA^{2}\right)$ & 1.9888 & 3.1531 & 3.8443 & 4.6194 \\
\hline
\end{tabular}

They represent the conditional expectation values of the polarization parameters at a given center-of-mass scattering angle.

In computation, the classical Hamilton's equations are numerically integrated in three dimensions. Four collision energies $\left(E_{c o l}=0.4,0.71 .0\right.$ and $\left.1.5 \mathrm{eV}\right)$ are chosen for stereodynamics calculation of the title reaction. The vibrational and rotational levels of $\mathrm{HI}$ molecule are taken as $v=0$ and $j=0$, respectively. $10^{5}$ trajectories are run for each initial collision condition and the integration step size is chosen as $0.1 \mathrm{fs}$. The trajectories started at an initial distance of $15.0 \AA$ between the $\mathrm{F}$ atom and the center of mass $(\mathrm{CM})$ of the HI molecule.

\section{Results and Discussion}

From table 1, we can see that both the maximum impact parameters and total reaction cross-sections increase with the increase of collision energies, which is in accord with Polanyi's propensity rule. ${ }^{23}$ Compared with the first reactive path, the second reactive path has smaller maximum impact parameters and total reaction cross-sections.

The $P\left(\theta_{r}\right)$ distributions describing the $\boldsymbol{k}-\boldsymbol{j}^{\prime}$ correlation are shown in figure $2 \mathrm{a}$. Obviously, the maximum of $P\left(\theta_{r}\right)$ is at $\theta_{r}=\pi / 2$ and symmetric with respect to $\theta_{r}=\pi / 2$, which indicates that $j^{\prime}$ is strongly aligned to the relative velocity direction at $\theta_{r}=\pi / 2$. In addition, the peak of $P\left(\theta_{r}\right)$ becomes slightly higher with the increase of collision energy, indicating that the degree of the product rotational alignment perpendicular to $\boldsymbol{k}$ becomes stronger. This is also confirmed by the value of the product rotational alignment parameters $\left\langle P_{2}\left(\cos ^{2} \theta_{r}\right)\right\rangle$, which are $-0.47919,-0.48596,-0.48895$ and -0.4916 corresponding to $\mathrm{E}_{\mathrm{col}}=0.4,0.7,1.0,1.5$ $\mathrm{eV}$, respectively. The closer the value of $\left\langle P_{2}\left(\cos ^{2} \theta_{r}\right)\right\rangle$ approaches to -0.5 , the stronger the $\boldsymbol{j}^{\prime}$ is aligned along the direction perpendicular to $\boldsymbol{k}$. In addition, due to HHL mass combination, the excessive translation energy will channel into rotational excitation, the product rotational angular momentum alignment is strong for four collision energies. 


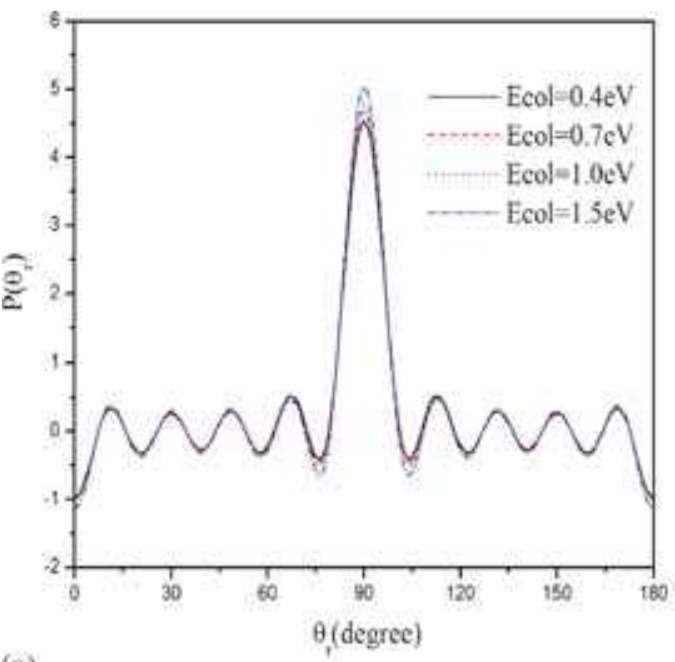

(a)

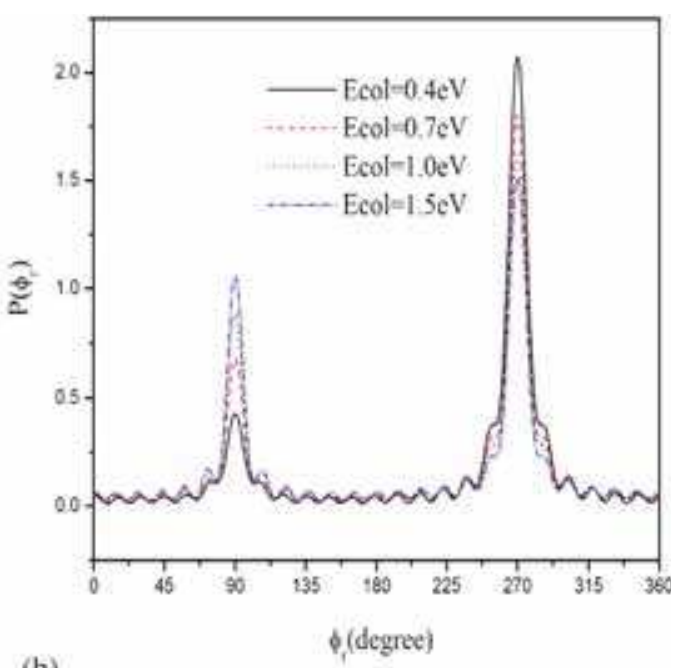

(b)

Figure 2. The $P\left(\theta_{r}\right)$ and $P\left(\phi_{r}\right)$ distributions at the four collision energies.

The dihedral angle distributions $P\left(\phi_{r}\right)$ shown in figure $2 \mathrm{~b}$ describe $\boldsymbol{k}-\boldsymbol{k}^{\prime}-\boldsymbol{j}^{\prime}$ correlations. The $P\left(\phi_{r}\right)$ distributions illustrate asymmetric properties with respect to the $\boldsymbol{k}-\boldsymbol{k}^{\prime}$ scattering plane (or about $\phi_{r}=\pi$ ). The distribution peaks appear at $\phi_{r}=\pi / 2$ and $3 \pi / 2$, indicating the angular momentum $j^{\prime}$ of the product FI is aligned along $\mathrm{y}$-axis of the $\mathrm{CM}$ frame. The peak at $\phi_{r}=3 \pi / 2$ is stronger than that at $\phi_{r}=\pi / 2$ for a fixed collision energy, which reveals that the $j^{\prime}$ of the product is oriented along the negative $y$-axis. Moreover, the orientation degree of angular momentum $j^{\prime}$ depends sensitively on the collision energy. As the collision energy

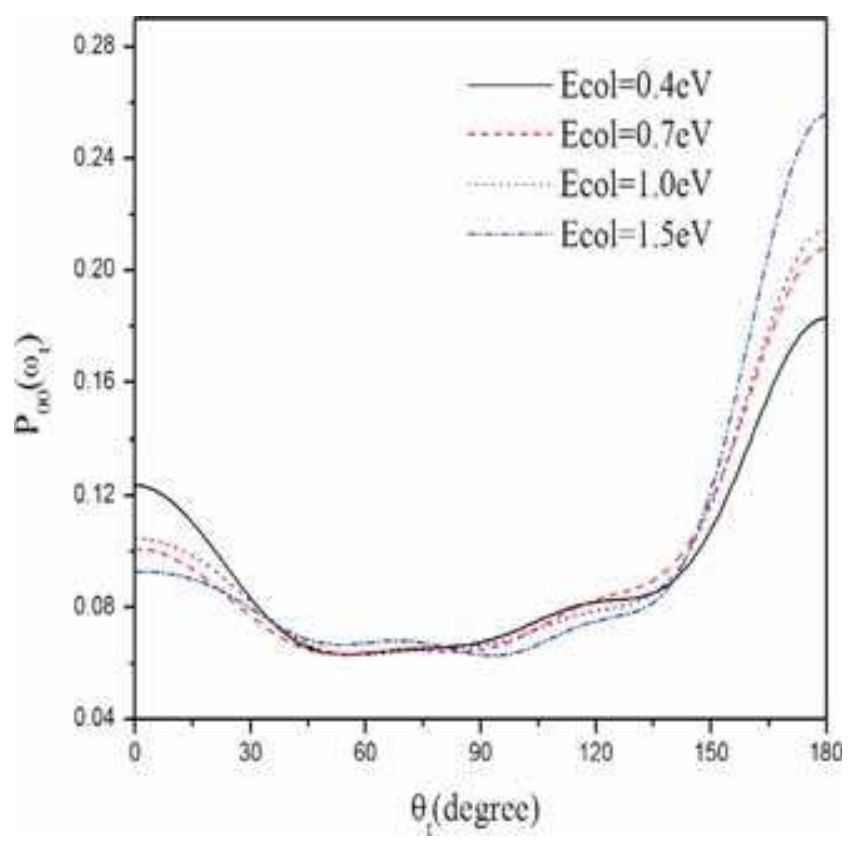

Figure 3. The $P_{00}\left(\omega_{t}\right)$ distribution at the four collision energies. increases, the peak at $\phi_{r}=\pi / 2$ becomes higher, while that at $\phi_{r}=3 \pi / 2$ becomes lower, that is to say, the orientation degree of angular momentum $j^{\prime}$ along the negative $y$-axis gradually weaken with the increase of collision energy. The thin distribution at $\phi_{r}=\pi / 2$ and $\phi_{r}=3 \pi / 2$ for all collision energies indicates that the reaction is mainly dominated by in-plane mechanism, in which the product molecule prefers rotating in the scattering plane. This asymmetric distribution of $P\left(\phi_{r}\right)$ can be explained by the repulsive model proposed by Han et al. ${ }^{24}$ In the chemical bond forming and breaking processes for the title reaction, the preference for the

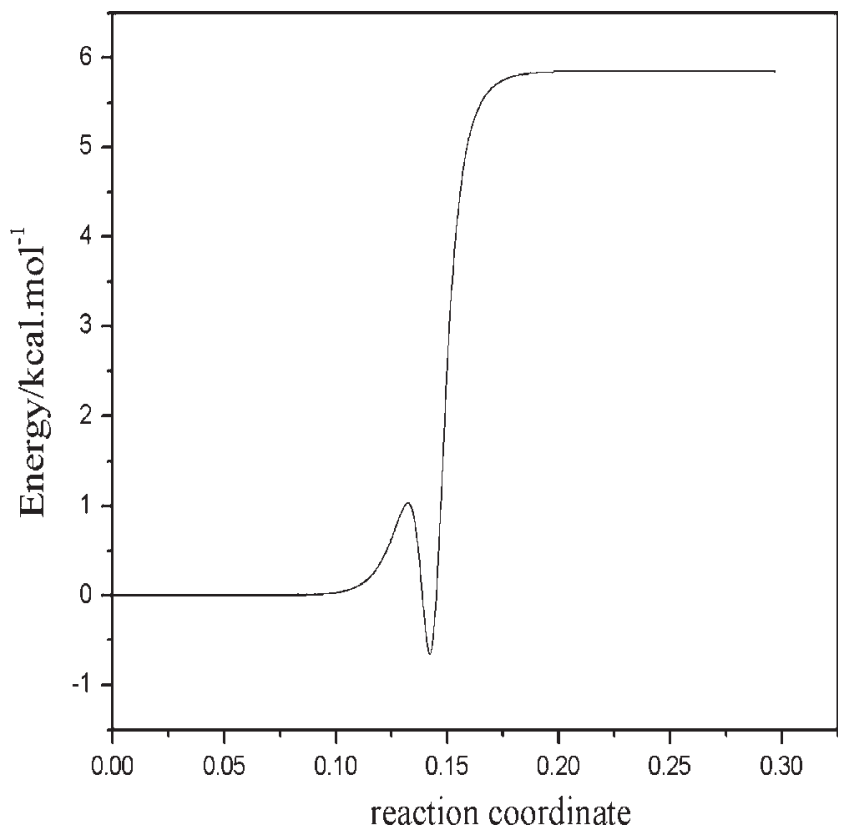

Figure 4. The reaction profile along the minimum energy path from reactants to products on the LEPS PES of the title reaction. 


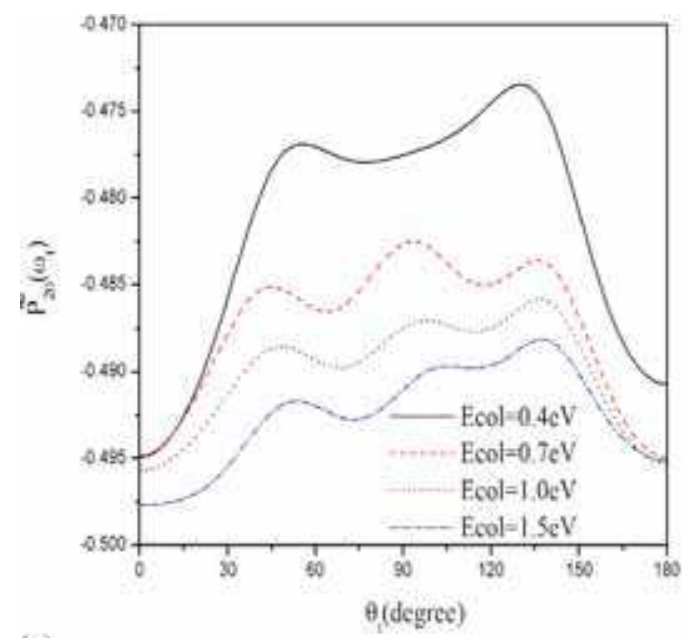

(a)

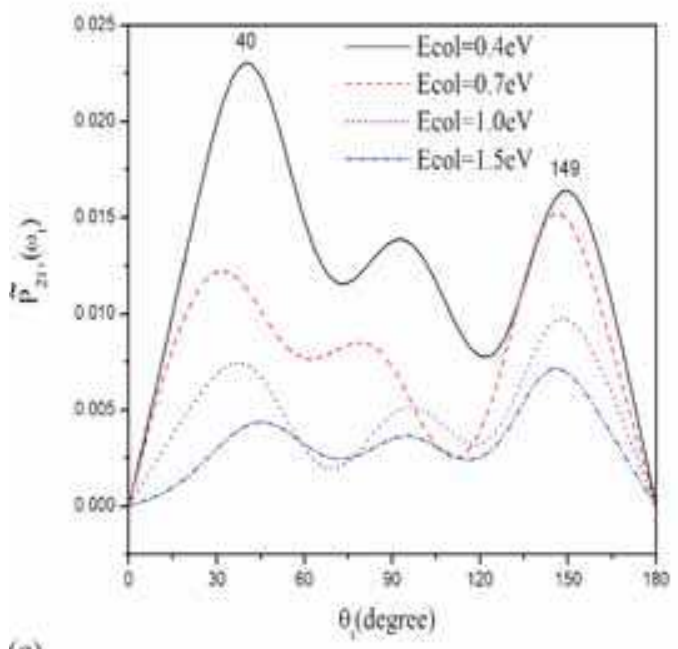

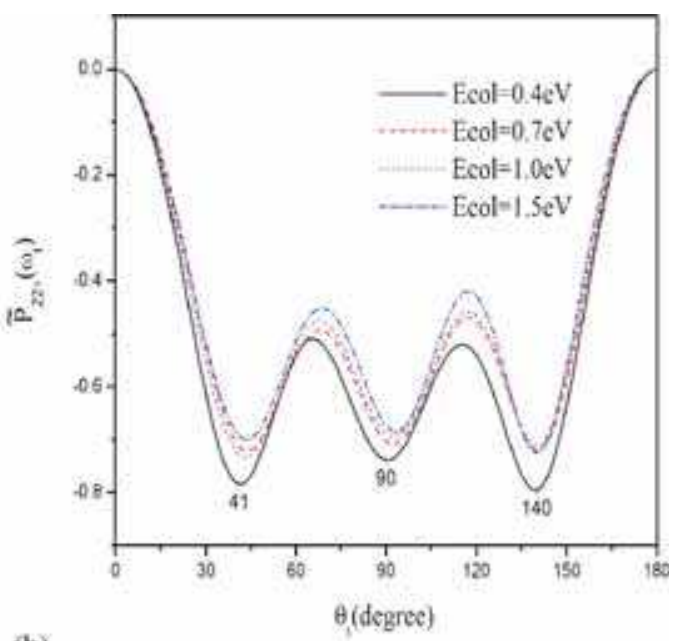

(b)

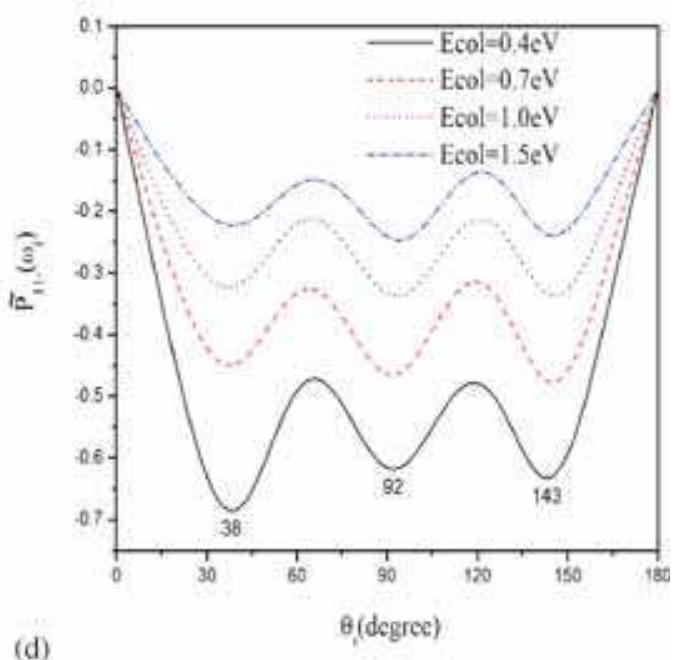

(d)

Figure 5. Four renormalized PDDCSs. (a) $\tilde{P}_{20}\left(\omega_{t}\right)$, (b) $\tilde{P}_{22+}\left(\omega_{t}\right)$, (c) $\tilde{P}_{21+}\left(\omega_{t}\right)$ and (d) $\tilde{P}_{11-}\left(\omega_{t}\right)$.

direction influenced by the repulsive energy between atom $\mathrm{I}$ and $\mathrm{H}$ will lead to the preferred orientation of the product's angular momentum.

The polarization-dependent generalized differential cross-sections (PDDCSs) describe the $\boldsymbol{k}-\boldsymbol{k}^{\prime}-\boldsymbol{j}^{\prime}$ correlation and the scattering direction of the product molecule. The $P_{00}\left(\omega_{t}\right)$ shown in figure 3 only describes the $\boldsymbol{k}-\boldsymbol{k}^{\prime}$ correlation or the scattering direction of the product. Figure 3 depicts that the product angular distribution is slightly away from the forward-backward symmetry, but peak in backward bias with the increase of collision energy, which can be ascribed to the property of the LEPS PES and impulse effect. As is shown in figure 4 , there exists a shallow potential well between two potential barriers in the reaction profile along the minimum energy path and the reaction is dominated by this insertion well at low collision energies. As the collision energy increases, a second higher barrier appearing on exit valley, whose height is about $6 \mathrm{kcal} / \mathrm{mol}$, plays an important role in the reaction dynamics. This repulsive barrier is late for this endothermic reaction, which makes the product molecule be preferentially scattered backward. The characteristics of these product angular distributions reflect an insertion dynamics for the title reaction. ${ }^{25}$ Similar results have also been found in reactions of $\mathrm{O}\left({ }^{1} \mathrm{D}\right), \mathrm{N}\left({ }^{2} \mathrm{D}\right), \mathrm{C}\left({ }^{1} \mathrm{D}\right)$, and $\mathrm{S}\left({ }^{1} \mathrm{D}\right)$ with $\mathrm{H}_{2}$ and its isotopomers. ${ }^{26}$

Figure 5 depicts several the angular distributions of the renormalized PDDCSs. The $\tilde{P}_{20}\left(\omega_{t}\right)$ distributions shown in Figure 5a are related to the expectation value $\left\langle P_{2}\left(\cos \theta_{r}\right)\right\rangle$. From figure $5 \mathrm{a}$, we can easily conclude that the product angular momentum $\boldsymbol{j}^{\prime}$ is preferentially polarized along the direction perpendicular to $\boldsymbol{k}$ at $\theta_{t}=0$ and $\pi$ for the four collision energies, and the $j^{\prime}$ tends to be aligned along the direction perpendicular to $\boldsymbol{k}$ with the increase of collision energies. The distributions of $\tilde{P}_{k q \pm}\left(\omega_{t}\right)$ with $q \neq 0$ are depicted in figures $5 \mathrm{~b}-5 \mathrm{~d}$. The collision energy has a considerable influence on these angular distributions. All $\tilde{P}_{k q \pm}\left(\omega_{t}\right)$ with $q \neq 0$ are equal to zero at $\theta_{t}=0$ and $\pi$. The $\tilde{P}_{22+}\left(\omega_{t}\right)$, 
$\tilde{P}_{21+}\left(\omega_{t}\right)$ and $\tilde{P}_{11-}\left(\omega_{t}\right)$ are relative to $\left\langle\sin ^{2} \theta_{r} \cos 2 \phi_{r}\right\rangle$, $\left\langle\cos \theta_{r} \sin \theta_{r} \sin \phi_{r}\right\rangle$ and $\left\langle\sin \theta_{r} \sin \phi_{r}\right\rangle$, respectively. The values of $\tilde{P}_{22+}\left(\omega_{t}\right)$ are negative for all scattering angle, as shown in figure $5 \mathrm{~b}$, indicating a marked preference of product alignment along the y-axis. In particular, the product displays a stronger polarization at about $40^{\circ}, 90^{\circ}$ and $140^{\circ}$. In figure $5 \mathrm{c}$, the values of $\tilde{P}_{21+}\left(\omega_{t}\right)$ are positive in all scattering angles, reflecting that $j^{\prime}$ is aligned along the direction of vector $\mathrm{x}+\mathrm{z}$. The absolute values of $\tilde{P}_{21+}\left(\omega_{t}\right)$ deviate from its maximum $\sqrt{3}$ far and away for the four collision energies because that $\boldsymbol{j}^{\prime}$ is preferentially polarized along the y-axis. The distribution of $\tilde{P}_{11-}\left(\omega_{t}\right)$ shown in figure $5 \mathrm{~d}$ describes the orientation of the product angular momentum $j^{\prime}$ along the y-axis. The $j^{\prime}$ is oriented along the negative direction of y-axis when the value of $\tilde{P}_{11-}\left(\omega_{t}\right)$ is negative, otherwise oriented along the positive direction of y-axis. It can be seen from the distributions of $\tilde{P}_{11-}\left(\omega_{t}\right)$ that $j^{\prime}$ is oriented along the negative direction of $y$-axis for four collision energies and the orientation degree of $\boldsymbol{j}^{\prime}$ weakens with the increment of collision energy, which is in accord with the distributions of $P\left(\phi_{r}\right)$.

\section{Conclusions}

In this paper, we have employed the quasiclassical trajectory method to study the effect of collision energies on stereodynamics of the reaction, $\mathrm{F}+\mathrm{HI} \rightarrow \mathrm{FI}+\mathrm{H}$. Several PDDCSs were calculated and the angle distribution curves of $P\left(\theta_{r}\right)$ and $P\left(\phi_{r}\right)$ at different collision energies are plotted. The calculated results show that the product rotational angular momentum $j^{\prime}$ is not only aligned but also orientated along the negative y-axis. As the collision energy increases, the alignment degree of products becomes slightly stronger, while the orientation degree of angular momentum $j^{\prime}$ along the negative $y$-axis gradually weakens. The collision energy has a considerable influence on the degree of polarization dependence of scattering angles. The title reaction can be governed by an insertion mechanism.

\section{Acknowledgement}

The author sincerely thanks Professor KeLi Han for providing the QCT Stereodynamics code. This work is by the National Natural Science Foundation of China (Grant 11205005).

\section{References}

1. Sung J P and Setser D W 1977 Chem. Phys. Lett. 48413

2. Beadle P, Dunn M R, Jonathan N B H, Liddy J P and Naylor J C 1978 J. Chem. Soc., Faraday Trans. 742170

3. Tamagake K, Setser D W and Sung J P 1980 J. Chem. Phys. 732203

4. Würzburg E and Houston P L 1980 J. Chem. Phys. 72 5915

5. Kornweitz H and Persky A 2004 J. Phys. Chem. A 108 140

6. Mu J X and Duan Z X 2009 J. Dalian. Jiao. Tong. University 30105

7. Ma J J and Cong S L Chin. J. Chem. Phys. 18319

8. Shafer-Ray N E, Orr-Ewing A J and Zare R N 1995 J. Phys. Chem. 997591

9. Aoiz F J, Bañares L and Herrero V J 1998 J. Chem. Soc. Faraday Trans. 942483

10. Aoiz F J, Brouard M and Enriquez P A 1996 J. Chem. Phys. 1054964

11. Miranda M P, Aoiz F J, Bañares L and Sáez Rábanos V 1999 J. Chem. Phys. 1115368

12. Brouard M, Gatenby S D, Joseph D M and Vallance C 2000 J. Chem. Phys. 1133162

13. Han K L, He G Z and Lou N Q 1996 J. Chem. Phys. 105 8699

14. Wang M L, Han K L and He G Z 1998 J. Chem. Phys. 1095446

15. Chen M D, Wang M L, Han K L and Ding S L 1999 Chem. Phys. Lett. 301303

16. Chen M D, Han K L and Lou N Q 2002 Chem. Phys. Lett. 357483

17. Chen M D, Han K L and Lou N Q 2003 J. Chem. Phys. 1184463

18. Miquel I, Hernando J, Sayós R and González M 2003 J. Chem. Phys. 11910040

19. Ma J J 2013 Acta Phys. Sin. 62023401

20. Ma J J, Chen M D, Cong S L and Han K L 2006 Chem. Phys. 327529

21. Ma J J, Zhang Z H and Cong S L 2006 Acta Phys. Chim. Sin. 22972

22. Wang Y H, Xiao C Y, Deng K M and Lu R F 2014 Chin. Phys. B 23043401

23. Ding A M G, Kirsch L J, Perry D S, Polanyi J C and Schreiber J L 1973 Faraday Discuss Chem. Soc. 55252

24. Han K L, He G Z and Lou N Q 1989 Chin. J. Chem. Phys. 2323

25. Yue X F, Cheng J, Li H, Zhang Y Q and Emilia L W 2010 Chin. Phys. B 19043401

26. Aoiz F J, Bañares L and Herrero V J 1996 J. Phys. Chem. A 11012546 